symptoms might indicate a serious illness; and were surprised at how long a RTI could persist for and how frequently a child could get a RTI, particularly in the first years of life. This was true for parents from all socio-economic backgrounds and with different levels of education. When consulting, parents were often seeking a medical assessment and reassurance. However, parents felt that clinicians were often dismissive and had not properly evaluated the child, often leading to re-consultations. In addition, clinician explanations of diagnosis and treatment recommendations were not well understood by parents, and they remained unclear about how to manage an RTI and when to consult.

Conclusion Parents' poor knowledge and un-realistic expectations in relation to RTIs in children contribute to high rates of consultation. Despite awareness of parental perspectives, there remains a problem with parent-HP communication in relation to key information needed by parents to manage child's illness confidently and know when to consult in future.

\section{OP66 A PROFILE OF UNDIAGNOSED DIABETICS IN THE COMMUNITY: RESULTS FROM THE BOSTON AREA COMMUNITY HEALTH (BACH) PRE-DIABETES SURVEY}

doi:10.1136/jech-2012-201753.066

RS Piccolo, JB McKinlay, LD Marceau. Health Services and Disparities Research, New UK Research Institutes, Watertown, USA

Background Research suggests almost $30 \%$ of the United States population has undiagnosed diabetes and that diabetics typically have the disease for 4 to 7 years before eventual diagnosis. Delay in initial diagnosis results in greater diabetes-related complications, poorer patient outcomes, and reduced quality of life. Most diabetes research is necessarily confined to diagnosed diabetes. Our objective was to identify risk factors for remaining undiagnosed.

Methods The Boston Area Community Health (BACH) Pre-Diabetes study is an ongoing community-based random-sample cohort study that has enrolled 2,974 participants to date. Participants were asked to fast for 8 hours prior to their interview. Fasting glucose (FG) and glycated hemoglobin (HbA1c) were collected during inhome interviews. Undiagnosed diabetes was defined as FG $>125$ $\mathrm{mg} / \mathrm{dL}$ or $\mathrm{HbA} 1 \mathrm{c} \geq 6.5 \%$. Risk factors were organized into four logical groupings: 1) socio-demographics, 2) lifestyle/behavioral, 3) utilization/access to healthcare, and 4) health status/comorbidities. Logistic regression was used to estimate the odds ratio (OR) for diagnosed vs. undiagnosed diabetes.

Results The prevalence of diabetes in the BACH study was $27.8 \%$ $(n=827) .21 .2 \%$ of diabetes cases were undiagnosed. The data indicate that healthcare utilization and the presence of co-morbid conditions had a large impact on diabetes diagnosis. Participants who visited a health care provider 5 or more times in the past year were $90 \%$ more likely to be diagnosed $(\mathrm{OR}=1.9, \mathrm{p}<0.001)$ than participants who had 5 or fewer visits. Participants with a history of high cholesterol were more than twice as likely to be diagnosed $(\mathrm{OR}=2.7$, $p<0.001)$. High blood pressure or a history of heart disease also increased the likelihood a participant was diagnosed. Participants with a documented family history of diabetes were more likely to be diagnosed $(\mathrm{OR}=2.9, \mathrm{p}<0.001)$. Finally, English-speaking participants were more than twice as likely to be diagnosed $(\mathrm{OR}=2.6, \mathrm{p}=0.01)$ than their Spanish-speaking counterparts.

Conclusion Undiagnosed diabetes is highly prevalent problem in the United States that leads to poorer patient outcomes and significant health-care costs. These results indicate that access to care, health care utilization and the presence of co-morbid conditions have an important impact on diabetes diagnosis. Individuals who do not have the traditional risk factors for diabetes (i.e. family history) have a greater risk of remaining undiagnosed. These findings suggest that improving access to care in the United States may greatly increase the likelihood of diagnosing previously undiagnosed diabetes.

\section{OP67 DO DOCTORS CONTRIBUTE TO THE SOCIAL PATTERNING OF DISEASE? CONTRASTING FINDINGS FROM AN EPIDEMIOLOGIC SURVEY AND A DECISION MAKING EXPERIMENT}

doi:10.1136/jech-2012-201753.067

J.B. McKinlay. Health Services and Disparities Research, New England Research Institutes, Watertown, USA

Background That type 2 diabetes mellitus (T2DM) varies significantly by race and ethnicity is a widely accepted fact. It is often invoked as a base rate (a priori probability) during the process of clinical reasoning. Epidemiological studies repeatedly show undiagnosed T2DM varies more by socioeconomic status (SES), than by race/ethnicity. This study seeks to understand the discrepancy between the true prevalence of undiagnosed T2DM by SES and its continually reported prevalence by race/ethnicity.

Methods Data from two different but complementary studies are employed: a) a large Boston Area Community Health (BACH) survey; and b) a factorial experiment conducted with primary care doctors to examine variations in clinical decision making. The $\mathrm{BACH}$ epidemiologic survey $(\mathrm{n}=5502)$ employed a stratified, multi-stage cluster sample design and used multivariable techniques including logistic regression. The factorial experiment concerning decision making employed clinically authentic videotaped scenarios presented to primary care doctors $(\mathrm{n}=192)$, and used ANCOVA analyses.

Results Results from the epidemiologic survey show that both undiagnosed signs and symptoms and diagnosed T2DM vary similarly by socio-economic status (SES). This finding is independently corroborated by National Health and Nutrition Examination Survey (NHANES) data for diagnosed T2DM. Complementary data from the clinical decision making experiment show the diagnosis of T2DM varies significantly by a patients' race/ethnicity, controlling for SES, age and gender in the design. While undiagnosed signs and symptoms of T2DM in the community vary significantly by SES, rather than race/ethnicity, following diagnosis by primary care doctors they vary more by race/ethnicity, rather than by SES.

Conclusion Race/ethnicity and SES in the US are almost totally confounded, such that measuring one is essentially also measuring the other. Consequently, doctors generally get the social patterning of T2DM right, but for entirely the wrong reason. Continued patterning of T2DM by race/ethnicity motivates the search for genetic and biophysiologic explanations and distracts attention from the more important and potentially modifiable contribution of SES circumstances to the prevalence of T2DM.

\section{OP68 THE CONTRIBUTION OF DOCTORS' DECISIONS TO HEALTHCARE INEQUALITIES: TOWARDS FOURTH GENERATION STUDIES}

doi:10.1136/jech-2012-201753.068

LD Marceau, JB McKinlay, RS Piccolo. Health Services and Disparities Research, New UK Research Institutes, Watertown, USA

Background Healthcare variations are a worrisome and well-documented problem. Such variations occur at different levels: between health care systems, geographic areas, organizational settings, patients, and doctors. Research is increasingly focused on the contribution of doctors' decision making to the generation or amplification of healthcare disparities. Clinical decision making studies continue to evolve through generations of work that focus on different types of influence: First generation studies identified patient-level attributes (e.g., gender, age, race/ethnicity, SES, health insurance status); Second generation studies on variations associated with physician characteristics (e.g., age, gender and education, specialty and type of remuneration); Third generation studies on health system and 
organizational influences (e.g., size, profit status, location and population served, and most recently, organizational culture).

Methods We used a factorial experimental design in which the subjects, primary care doctors $(\mathrm{n}=192)$, viewed clinically authentic vignettes of "patients" presenting with identical signs and symptoms suggesting diabetes. They were stratified according to gender and level of experience. During an in-person interview, they were asked how they would diagnosis and manage the vignette 'patient'. Results After controlling for the first two levels of influence (patient and provider), each of which contributed 4.4 and $2 \%$ respectively, organizational culture significantly contributed to their behavior, accounting for $14.3 \%$ of the variation in clinical decision-making for diabetes. Considering nine different dimensions of practice culture, organizational trust and business emphasis contributed most to the variance in treatment for diabetic foot neuropathy.

Conclusion Attempts to reduce health care variations continues to focus on the levels of patient attributes and physician characteristics (e.g. improved educational efforts). Findings from this experiment suggest a need to further investigate the contribution of of organizational factors (third generation) and suggest appropriate interventions at that level. As important however, is the consideration of a fourth generation: cognitive aspects of physician decisions. Qualitative methods (i.e. "think aloud") and newly developing methods to objectively measure unconscious bias (e.g., the Implicit Association Test) can provide more robust assessment of what goes on "inside the doctors' head," which eventually produce healthcare disparities.

\section{HSR: General}

\section{OP69 THE USE OF NHS ROUTINE DATA TO ANALYSE THE EFFECTS OF HEALTHCARE INTERVENTIONS}

\author{
doi:10.1136/jech-2012-201753.069
}

'E V Cecil, 'M Soljak, ${ }^{2 D}$ Osipovic, ${ }^{2}$ S Peckham, ${ }^{1}$ A Majeed. 'Primary Care and Public Health, Imperial College, London, UK; ${ }^{2}$ Health Services Research and Policy, LSHTM, London, UK

Background In 2007, the strategy 'Healthcare for London' planned the establishment of polyclinics in each PCT to help improve the primary care infrastructure. These polyclinics serve as a hub for a group of GP practices which combined form a polysystem. We aimed to investigate the impact of services provided in a polysystem on unplanned (emergency) admissions, using routine NHS activity data. In one polysystem case study, a pulmonary rehabilitation service for COPD was established. In another, a diabetes patient education programme was offered.

Methods We used routine Hospital Episodes Statistics inpatient data from $2004 / 5$ to 2009/10 covering around 1500 London practices. Differences in adjusted trends in emergency admission rates were compared between the polysystem GP practices case studies and other London practices. Multilevel regression models assessed standardized rate ratios, controlling for year variation, GP practice and population factors. We incorporated interaction terms to allow the effects of the polysystem to vary independently by year.

Results Over the study period COPD emergency admission rates fell across London with a yearly admission rate ratio of (95\% CI) $0.98(0.97,0.99, \mathrm{p}<0.001)$. The rates in the study polysystem did not differ from the rest of London at baseline and there was not enough evidence to suggest that the introduction of the polysystem service in May 2007 had any effect on the admission rates. A total of $12 \%$ of the registered COPD population was seen as new contacts in the first year of the service.

Diabetes emergency admission rates have been falling across London over the study period with a yearly admission rate ratio $(95 \%$ CI) of $0.98(0.96,0.99, p=0.001)$. In the first year of the diabetes intervention, the rate of emergency admissions for diabetes fell by $80 \%$ in patients from the polysystem practices compared with London, with an interaction factor $(95 \%$ CI) of $0.20(0.13,0.31) \mathrm{p}<0.001$ and this fall was maintained in the following year. Intervention in this London Polysystem covered $70 \%$ of the diabetic population. The power to detect an effect was increased due to the coverage.

Conclusion NHS routine data can be used to assess the impact of health service interventions that are aimed at reducing admissions. Commissioners must be aware that to assess the impact of interventions, the implementation needs to be on a large scale and that medium term follow up is required in order to study the trends.

\section{OP70 PLACE OF CANCER DEATHS IN ENGLAND, 2001-2010: TIME TRENDS AND DETERMINANTS}

doi:10.1136/jech-2012-201753.070

'W Gao, 'Y Ho, ${ }^{2} \mathrm{~J}$ Verne, ${ }^{3} \mathrm{M}$ Glickman, 'IJ Higginson. 'Palliative Care, Policy and Rehabilitation, King's College London, London, UK; ${ }^{2}$ South West Public Health Observatory, Bristol, UK; ${ }^{3}$ Health and Life Events Division, Office for National Statistics, Newport, UK

Background Place of death is an important quality indicator for end of life care. Understanding where patients with cancer die and what factors are associated with place of death is important for improving end of life care and has significant health care cost implications. There is, however, limited up-to-date evidence to guide practice.

Methods This is a whole population-based observational study. All deaths with cancer as the underlying cause of death (ICD-10 codes: C00-C97), which occurred in the period 2001-2010, were extracted from death registrations in England. The time trends in place of death (coded as hospital, home, hospice, nursing home and elsewhere) were described and tested by using the Cochran-Armitage Trend Test. Factors associated with hospital death (age, gender, year of death, cancer site, index of multiple deprivation (IMD), region) were evaluated using the generalised linear model. The clustering effect within region was accounted for using an exchangeable working correlation structure.

Results A total of 1,270, 071 cancer deaths were recorded over the 10 years period, of which $47.4 \%$ occurred in hospitals, $24.1 \%$ in homes, $17.1 \%$ in hospices, $6.6 \%$ in nursing homes and $4.8 \%$ elsewhere. In the study period, deaths in hospital decreased from $49.7 \%$ to $42.2 \%$ (annual decrease $0.8 \% ; \mathrm{P}_{z}={ }_{59.6}<0.001$ ), and home deaths increased from $22.3 \%$ to $27.6 \%$ (annual increase $0.4 \% ; \mathrm{P}_{z}={ }_{48.7}<0.0001$ ). Independent risk factors for dying in hospitals were: being older (Adjusted $\mathrm{OR}_{75-84 \text { vs 18-54 }}$ (AOR): $1.25 ; 95 \%$ confidence interval (95\%CI): 1.24-1.26) and living in deprived areas (AORs:1.06-1.31). Leukaemia $\left(\mathrm{AOR}_{\mathrm{ref}}=\right.$ : 3.66 ; 95\%CI: 3.65-3.68), Non-Hodgkin's Lymphoma $\left(\mathrm{AOR}_{\mathrm{ref}}=u_{\text {lun: }}: 2.89 ; 95 \% \mathrm{CI}: 2.88-2.90\right)$ and Bladder cancer $\left(\mathrm{AOR}_{\mathrm{ref}}=1.46\right.$; 95\%CI: 1.45-1.47) were the top three cancer deaths most likely to occur in hospitals. Men had a slightly higher chance than women of dying in hospitals (AOR: 1.13; 95\%CI: 1.13-1.14). Cancer deaths in hospitals were more likely to occur in London than in South West (AORs range: 0.84 in South East Coast to 1.27 in London).

Conclusion We found an overall reducing trend in hospital deaths and an increasing trend in home deaths in the past ten years. However, significant inequality in place of death still exists. Future research needs to explore the underlying reasons.

\section{OP71 DISCRIMINATING CLINICAL OUTCOME MODELS MAY DRIFT UNACCEPTABLY: EXAMPLE OF CARDIAC SURGERY MORTALITY}

doi:10.1136/jech-2012-201753.071

${ }^{1} \mathrm{~K}$ McAllister, ${ }^{1} \mathrm{G}$ Hickey, ${ }^{1,2} \mathrm{~S}$ Grant, ${ }^{1,2} \mathrm{~B}$ Bridgewater, 'I Buchan. 'Northwest Institute for Biohealth Informatics, University of Manchester, Manchester, UK; ${ }^{2}$ Department of Cardiac Surgery, University Hospital of South Manchester, Manchester, UK 